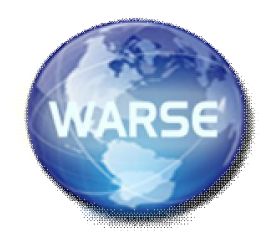

Volume 9, No.1, January - February 2020

International Journal of Information Systems and Computer Sciences

Available Online at http://warse.org/IJISCS/static/pdf/file/ijiscs01912020.pdf

https://doi.org/10.30534/ijiscs/2020/01912020

\title{
Decrease Electricity Consumption in Rooms with IoT Technology
}

\author{
Amir Seyyedabbasi ${ }^{1}$, Farzad Kiani ${ }^{2}$ \\ ${ }^{1}$ Computer Engineering Dept., Engineering and Natural Sciences Faculty, Istanbul Sabahattin Zaim University, \\ Istanbul, Turkey \\ amir.seyedabbasi@gmail.com \\ ${ }^{2}$ Computer Engineering Department, Engineering and Architecture Faculty, Istanbul Arel University \\ farzad.kiyani@gmail.com
}

\begin{abstract}
In this paper, we present a new IoT system to control electricity consumption in homes. The main goal of the proposed system is smart homes and devices. IoT systems also benefit sensor nodes and other heterogeneous things to work cooperatively. Wireless sensor network also has the main effect in the IoT projects. In the Proposed system, there are different sensors to control temperature, the light of rooms. Besides actuators to perform commands. There is an application to monitor and manage the room events and send notifications when is an event established. The main motivation for this study to balance energy consumption. In the proposed system, also there is a motion sensor to send events at an appropriate time. Also, the weather forecast API is used in the application to perform on some operations based on weather conditions. The proposed IoT system is suitable for monitoring and managing homes and energy consumption.
\end{abstract}

Key words: IoT, smart room, monitor home, energy efficiency, electricity consumption.

\section{INTRODUCTION}

The advances in technology and internet technologies help the human to control daily life. During the last years, most of the electrical devices in the home increased [1]. Each of these devices has a different energy consumption rate. Some devices are the necessity for human life such as refrigerator, oven, and washing machine and so on. The energy reserves are decreasing according to last researches [2]. The internet of things (IoT) systems are being the inseparable unit of our life by growing technology and the internet. The interaction of home devices via the internet has made it easy to manage these devices.

The main feature of IoT is the integration of different devices with each other. Consider, the lights can be controlled via an application. There are three different units the light, application, and actuator. This leads to intelligent systems in the home. Also, the intelligent systems have some challenges although new opportunities to become smarter [3]. The IoT systems, collect multiple information via sensors. The actuators perform a task when it gets a command. IoT system is also applied in many areas such as agriculture [4], health [5], traffic [6], and industry [7]. The Experiments with IoT and WSN encouraged us to develop a smart room [4]. The proposed method is designed to control energy consumption. In most of the country like European and Asians countries, the energy cost is very high. In this work, we focused on electric energy consumption.

This study by creating a smart room helps users to manage room temperature and luminosity. In the classic homes heating facilities are used to increase the temperature of the room normally, although radiators can be controlled by a handle from minimum to maximum, it is manually. In this proposed system, no human intervention in the regulation of room temperature. The human can set radiators in maximum, but the combi boiler will set room temperature. The proposed system has a temperature sensor in the room to control room temperature. In the application, the human can define the configurations to control radiators.

Also, lamps are one of electric consumption devices. Although, the new generation of lights are energy efficient. However, the proposed IoT system control electricity consumption. Classic lamps in different cases can switch to turn off/on. In the proposed system the smart lamps can control via the application. When the person forgot the turn-off lights in leaving home, the application sends the notification. Also, there is a motion detection sensor in the room. This sensor control luminosity in the room.

IoT based on the internet and heterogeneous things collect different information, besides the actuators perform the commands [8]. The proposed system is suitable for each person which familiar with the beginnings of the internet. With the advent of IoT technology, millions of devices including cars, lights, water systems, and cell phones will be connected. IoT has some challenges because each event from things records in the storage. The security of this data is an issue [9]. The rest of the paper is structured as follows; the second section provides the related works in smart rooms and 
homes. The third section describes the proposed IoT system. Finally, the conclusion and future work of this study.

\section{RELATED WORKS}

This section describes some researchers about smart home and rooms, in most studies, the authors' tries to find a way to increase human interference in the homes. In [9], the authors proposed a new IoT system to count and people participating in the ceremonies. They proposed a smart room, detects motions to count and track peoples. In this way, they used also a low-resolution camera. In [10], the authors try to manage rooms' lights, cooling, heating, and projector operations. They also, proposed this method to help conferences in the smart rooms. They focused on the main operations in the meeting rooms such as control lights, humidity, and temperature. Besides, the application gave end-user a friendly user interface to control and manage rooms fan coils to the circulation of the weather. Authors in the [11] proposed an IoT system for campuses. In this system, they propose a smart room, smart parking and smart education schemes, which integrated with student portals. This system suggests available parking lots, classrooms for the students. They used some sensors such as PIR, RFID, and camera to give information to students. In the [12] authors proposed a new IoT system for motion detection in rooms. In their system, there are Raspberry Pi to collect the rooms' information. Also, there is cloud storage to send recordings on the cloud server. If in the room motion is detected the proposed system sends an email to admin to report the detected event. Generally, there a camera that connects with Raspberry Pi. It also communicates via TCP/IP protocol with an FTP server or cloud storage.

Most of the studies in the smart room in the literature used some sensors to achieve the IoT system. In this paper, we also propose a new IoT smart room with some sensors to achieve efficient usage of electricity. This helps peoples to manage cost and bedside saving energy reserves to futures.

\section{PROPOSED IOT SYSTEM}

In the proposed system, there are some sensor nodes in the room to control energy consumption. The IoT mechanism for this room consists of electrical and sensing units. As shown in figure 1 , there are two windows, two smart lights and two radiators in this room. On the other hand, there are six sensor nodes to measure the temperature and light level in the room and a motion detection sensor. Also, there is a user-friendly application to record the events in the room. Besides the end-user can define some scenarios from this application to the related room.

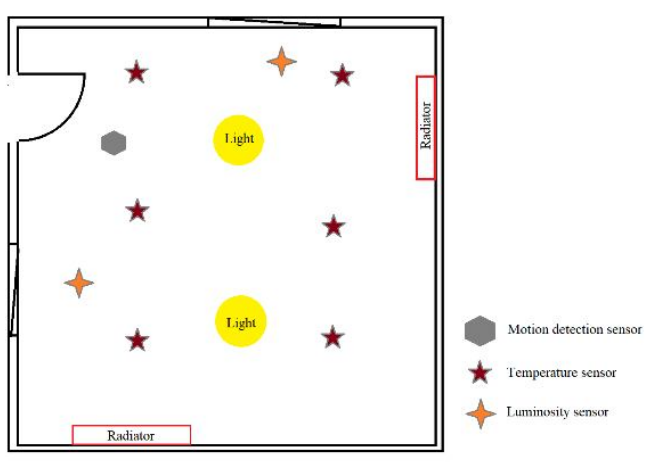

Figure 1: The proposed method room architecture.

The first important step in the IoT systems is data collection. This is done with sensor nodes, RFID devices or DCs. In the proposed system the five sensor nodes collect and acquire data from the room. Wireless sensor nodes are the main units of IoT systems. The sensor nodes collect raw data, so the necessity of application is determined. The sensor nodes are some things of IoT systems.

After data collecting, the next step is recording. The second step is the storage of the collected data. In most of the sensor nodes, there is a storage unit, but it is not efficient. In the IoT system maybe there are more than one year events data. In this way, in the most IoT systems, the data will be stored in the external storage or in the server.

Analyzing is the third step of IoT systems. In this step, the raw data should be processed. In most IoT system there is an application to interact with a human to control and monitor events. This is special for each application. As well as, the application is the interface with end-user and things in IoT systems.

The fourth step of IoT systems performs a task. The actuators perform something based on the pre-defined scenarios. The actuator may be a sensor node or an electrical device. In the proposed method, the actuators are smart lights and combi boiler.

\section{System architecture}

Wireless sensor networks (WSN) consists of a group of sensor nodes that composed and collect and transfer area detected events to the base station (BS). Sensor nodes in the deployed area detect phenomena. Based on the application the event detected and as a data packet transferred to the server. Most of the nodes equipped with different sensors. Also, each sensor node has four main units such as power, Computing, sensing and communication units. One of the main issues of the sensor node is power limitation. Besides, the ease of use and easy to establish the advantages of sensor nodes [13]. 


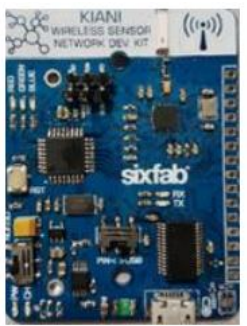

Figure 2: KIANI sensor node

The sensor nodes that we used in the proposed method are KIANI sensor nodes [4]. The communication unit of sensor nodes equipped with Texas instruments cc1101 Low-Power Sub-1GHz RF Transceiver. The computing unit is also equipped with Arduino Nano, ATmega328P. The power unit has a rechargeable battery. Lithium-ion battery is $1200 \mathrm{mah}$ 3.7v Li-ion. Furthermore, in the sensing unit, there are three different sensors. The first one is the temperature sensor that physically embedded in the KIANI sensor to measure the temperature of the room. The BH1750 light sensor is added to the KIANI sensor nodes to measure the luminosity of the room. These sensor nodes are one unit of the proposed IoT system. Also, the motion detection sensor is equipped for these sensor nodes.

The other part of the IoT system is a gateway, RASPBERRY PI 3 is used to collect sensed data from sensor nodes. Each sensor node communicates with the gateway as a base station. The base station transferred the data packet to the application via the TCP/IP protocol. In this way, the end-user can monitor the room temperature and light level. Nowadays, in most developing countries, humans do not spend more time in the home. The end-user via mobile application or website can manage energy consumption. As an example, in the winter days, when a person leaves home at 7 am and came back at 6 $\mathrm{pm}$, they want to go home with a high temperature. Most parents leave home very early; the baby wakes up later than them babes will be scared when wakes up in the darkroom. Based on this application, the end-user increases the room temperature and turn on lights for babies. As mentioned above, there are two actuators in this system, smart lights, and Combi boiler. Each of the smart lights equipped with switchers to control off and on the Combi boiler too. The switchers are communicated with the gateway via Bluetooth. In the proposed system, each actuation takes place in real-time and automatically. In this system, the end-user monitor rooms events. Also, they can define some scenarios for hours and days. This is aimed to satisfy the end-user needs also. The third sensor that the proposed system is equipped with is motion detection. Consider, a day that the person goes shopping and bought most of the things, enters the room and can't find the light keys to turn on lights. In this time motion detection sensor detect a person entrance and turn on light if the end-user active this.

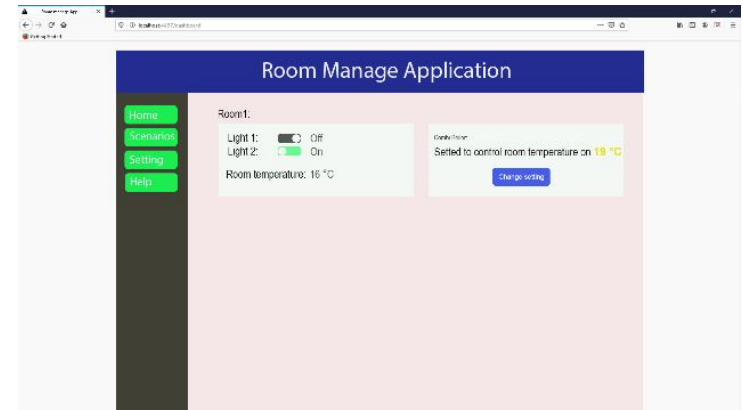

Figure 3: Screenshot of proposed system application

This proposed system helps people to manage electricity consumption. Another assistance of this system is to monitor energy consumption for combi boilers. Also, this system benefits a user-friendly interface. The application is also can send a notification when the room temperature is higher than the default. The application gets the city weather forecast from weather webservers API to control the home temperature. Besides, send messages to the end-user when forgot to turn off the light when daylight is enough. The application is also can synchronized with the person trips calendar. Based on the end-user default definition all these options help the person decrease electricity consumption.

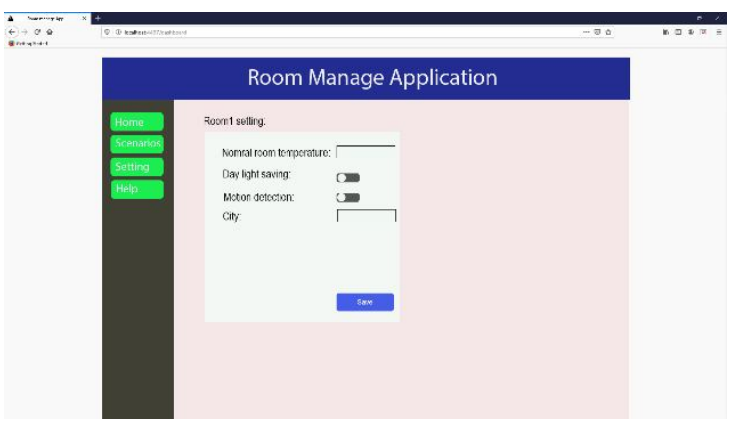

Figure 4: Screenshot of proposed system application.

Generally, the IoT systems have three components such as hardware, software and cloud service. The proposed system is also having these three units. The rooms' events and information are also uploaded to the google drive of the person google account. The application can upload and download these events when needed.

\section{CONCLUSION}

Energy consumption is one of the issues nowadays. Energy and gas reserves will be finished at the end. The energy-saving is one of the current century duties. The technology and the internet can be helping people to reduce energy consumption. The number of machines and devices is growing in the world and internet usage proves as a need of the current century. Internet of things (IoT) is a new technology that connects every device via the internet to each other. Each device that connected to the internet can be a thing in IoT projects. One of the important things in IoT projects is sensors. The sensor also helps to IoT to collect information from the area and transfer to the servers. There is a user interface to the interaction between human and IoT 
systems. The application allows the end-user to monitor events on the area and define some scenarios in the application.

In the proposed IoT system, the main goal is to reduce electricity consumption. We suppose there is a room with some sensors, lights, and radiators. the sensors measure room temperature, light level, and motion detection. Each detected information will be sent to the server via the RASPBERRY PI 3 gateway. The application allows the end-user to define some scenarios (definition) in the room. Based on the simulation there is a significant decrease in energy consumption. Especially, the radiator consumption has more effect on electricity consumption. Also, this application is reliable due to all the information from rooms is actual and no human interference.

As future work, electricity consumption can be planned for a large home with more electronic devices. Also, focus on the automatic complex systems. Customize the GUI for the specific usage such as in manufactures and homes. Besides, will extend this project to some devices in the manufactures.

\section{REFERENCES}

1. D. Miorandi, S.Sicari, F. De Pellegrini, \& I. Chlamtac. Internet of things: Vision, applications and research challenges. Ad hoc networks, 10(7), 1497-1516, 2012. https://doi.org/10.1016/j.adhoc.2012.02.016

2. S. Shafiee, \& E. Topal. When will fossil fuel reserves be diminished?. Energy policy, 37(1), 181-189, 2009.

3. A. Al-Fuqaha, M. Guizani, M. Mohammadi, M. Aledhari, \& M. Ayyash. Internet of things: A survey on enabling technologies, protocols, and applications. IEEE communications surveys \& tutorials, 17(4), 2347-2376, 2015.

4. F. Kiani, A. Seyyedabbasi . Wireless sensor network and internet of things in precision agriculture. Int. J. Adv. Comput. Sci. Appl, 9(8), 220-226, 2018.

5. H. Hamidi. An approach to develop the smart health using Internet of Things and authentication based on biometric technology. Future generation computer systems, 91, 434-449, 2019.

https://doi.org/10.1016/j.future.2018.09.024

6. M. Vardhana, N. Arunkumar, E. Abdulhay, P.V. Vishnuprasad. Iot based real time trafic control using cloud computing. Cluster Computing, 22(1), 2495-2504, 2019.

7. W.Z. Khan, M.H. Rehman, H.M. Zangoti, M.K. Afzal, N. Armi, \& K. Salah. Industrial internet of things: Recent advances, enabling technologies and open challenges. Computers \& Electrical Engineering, 81, 106522, 2020.

8. A. Darwish, A.E.Hassanien, M. Elhoseny, A.K. Sangaiah, \& K. Muhammad. The impact of the hybrid platform of internet of things and cloud computing on healthcare systems: opportunities, challenges, and open problems. Journal of Ambient Intelligence and Humanized Computing, 10(10), 4151-4166, 2019. https://doi.org/10.1007/s12652-017-0659-1

9. D. Sgouropoulos, E. Spyrou, G. Siantikos, \& T. Giannakopoulos. Counting and tracking people in a smart room: An IoT approach. In 2015 10th international workshop on semantic and social media adaptation and personalization (SMAP) (pp. 1-5). IEEE, Nov 2015.
10. G. Sfikas, C.Akasiadis, E. Spyrou. Creating a Smart Room using an IoT approach. In Proceedings of the Workshop on AI and IoT (AI-IoT), 9th Hellenic Conference on Artificial Intelligence, Thessaloniki, Greece (pp. 18-20), May.2016.

11. M. W.Sari, P. W.Ciptadi, \& R.H. Hardyanto. Study of smart campus development using internet of things technology. In IOP Conference Series: Materials Science and Engineering (Vol. 190, No. 1, p. 012032). IOP Publishing, April, 2017.

12. A. N. Ansari, M. Sedky, N. Sharma, \& A.Tyagi . An Internet of things approach for motion detection using Raspberry Pi. In Proceedings of 2015 International Conference on Intelligent Computing and Internet of Things (pp. 131-134). IEEE, January 2015.

13. H. Suo, J.Wan, L.Huang, \& C. Zou. Issues and challenges of wireless sensor networks localization in emerging applications. In 2012 International Conference on Computer Science and Electronics Engineering (Vol. 3, pp. 447-451). IEEE, March 2012.

https://doi.org/10.1109/ICCSEE.2012.44 